\title{
Comparison between observed and theoretical O IV line ratios in the UV/EUV solar spectrum as derived by SUMER, CDS and EIS
}

\author{
A. S. Giunta ${ }^{1,2}$, A. Fludra ${ }^{2}$, M. G. O’Mullane ${ }^{1}$, and H. P. Summers ${ }^{1}$ \\ 1 Department of Physics, University of Strathclyde, 107 Rottenrow, Glasgow G4 0NG, UK \\ e-mail: alessandra.giunta@stfc.ac.uk \\ 2 STFC Rutherford Appleton Laboratory, Chilton, Didcot, Oxon. OX11 0QX, UK \\ Received 29 September 2011 / Accepted 12 December 2011
}

\section{ABSTRACT}

\begin{abstract}
Aims. The joint use of SoHO Solar Ultraviolet Measurement of Emitted Radiation (SUMER), Coronal Diagnostic Spectrometer (CDS) and Hinode Extreme-ultraviolet Imaging Spectrometer (EIS) allow observation of several O Iv line ratios, useful for temperature plasma diagnostics. Accurate atomic data are required to avoid interpretation errors in deriving the electron temperature from these ratios. Muglach et al. (2010) found that the measured ratio $I(787.72 \AA) / I(279.93 \AA)$ is lower than the predicted value by a factor $2-5$. Here the predicted value for this ratio is revised using updated atomic data. A comparison with other observed $\mathrm{O}$ IV line ratios is shown and the electron temperature is derived.

Methods. The analysis is based on new observations made during the observational campaign of April 2009 and including three $\mathrm{O}$ Iv multiplets. The theoretical ratios have been derived using the Atomic Data and Analysis Structure (ADAS) database and include comparison with the most recent calculations available in the literature.

Results. The discrepancy for the O IV $I(787.72 \AA) / I(279.93 \AA)$ ratio has been solved by adding transitions involving higher excited levels, which have been omitted in previous atomic models. This results in a decrease of the theoretical line ratio, providing electron temperatures in the range of $\log T=5.17-5.39$, close to the temperature expected from a plasma in ionisation equilibrium.
\end{abstract}

Key words. techniques: spectroscopic - Sun: transition region - Sun: UV radiation - Sun: atmosphere - atomic data

\section{Introduction}

The wavelength ranges of SoHO/SUMER, SoHO/CDS and Hinode/EIS contain several lines arising from the $\mathrm{O}^{3+}$ ion which are useful for temperature diagnostics and free from blends. SUMER allows observation of the $\mathrm{O}$ iv lines which belong to the $2 s^{2} 2 p^{2} P-2 s 2 p^{2} D$ multiplet: $2 s^{2} 2 p^{2} P_{1 / 2}-2 s 2 p^{2} D_{3 / 2}$ gives rise to $\mathrm{O}$ Iv $787.72 \AA$ while $2 \mathrm{~s}^{2} 2 \mathrm{p}^{2} \mathrm{P}_{3 / 2}-2 \mathrm{~s} 2 \mathrm{p}^{2}{ }^{2} \mathrm{D}_{3 / 2}$ and $2 s^{2} 2 p^{2} P_{3 / 2}-2 s 2 p^{2}{ }^{2} D_{5 / 2}$ are a self-blend of the lines at $790.11 \AA$ and $790.19 \AA$ observed at about 790.1 $\AA$. CDS observes the $2 \mathrm{~s}^{2} 2 \mathrm{p}^{2} \mathrm{P}-2 \mathrm{~s} 2 \mathrm{p}^{2} \mathrm{P}$ multiplet, whose four components (at $553.33 \AA, 554.08 \AA, 554.51 \AA$ and $555.76 \AA$ ) can be resolved by an appropriate fitting procedure. Finally, EIS observations include the two lines at $279.63 \AA$ and $279.93 \AA$, which arise from the $2 \mathrm{~s}^{2} 2 \mathrm{p}^{2} \mathrm{P}_{1 / 2,3 / 2}-2 \mathrm{~s}^{2} 3 \mathrm{~s}^{2} \mathrm{~S}_{1 / 2}$ transitions. The schematic partial level diagram in Fig. 1 illustrates the three line sets observed by SUMER, CDS and EIS, with the corresponding transitions and energy levels, and Table 1 lists the spectral lines with the respective instruments.

These three line sets provide several line ratios which are sensitive to the electron temperature of the plasma from which the lines are emitted. The ratio between the SUMER line at $787.72 \AA$ and the EIS line at $279.93 \AA$ has been examined recently by Muglach et al. (2010), who analysed data taken during a joint SUMER-EIS campaign in April 2007. They selected spectra obtained in quiet regions on the solar disk, but which included some small-scale coronal bright points. The theoretical ratio was obtained using version 5.2.1 of the CHIANTI database
(Landi et al. 2006), but we note that the current CHIANTI version (6.0.1, Dere et al. 2009) ${ }^{1}$ does not modify the O Iv data used for examination of this line ratio. Muglach et al. (2010) performed calculations at three different electron densities, $N_{\mathrm{e}}=$ $10^{9}, 10^{10}$ and $10^{11} \mathrm{~cm}^{-3}$, where they found that the measured ratio in all cases was smaller by a factor 2-5 than the theoretical ratio. From these measurements they derived an electron temperature in the range of $\log T=5.58-6.07$ (where the temperature is measured in Kelvin). Such temperatures are much higher than the temperature of maximum fractional abundance, $T_{\mathrm{m}}$, in ionisation equilibrium for O Iv. Muglach et al. (2010) adopted the value $\log T_{\mathrm{m}}=5.24$ based on calculations of Mazzotta et al. (1998). In the present work the value $\log T_{\mathrm{m}}=5.11$ at $N_{\mathrm{e}}=10^{10} \mathrm{~cm}^{-3}$ has been taken from the ADAS database (Summers 2001).

To explain the anomalous behaviour of the $I(787.72 \AA)$ / I(279.93 $\AA$ ) ratio, Muglach et al. (2010) investigated various possible causes, ranging from instrumental and observational effects to the presence of additional processes such as photoexcitation from photospheric black body radiation, self-absorption and non-Maxwellian distribution of the free electrons. However, they did not resolve the discrepancy, with the implication that a non-equilibrium ionisation state may be present. In this paper, atomic data for the $\mathrm{O}$ iv lines are revisited in detail. The ratio

1 The impending CHIANTI version 7.0 also does not change the situation. 
A\&A 538, A88 (2012)

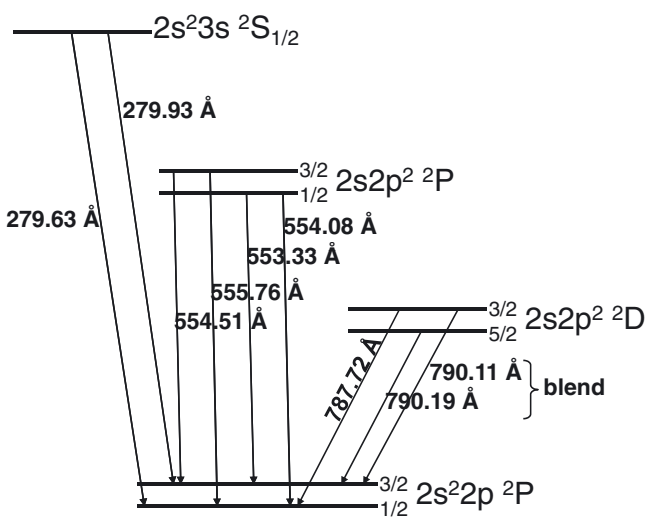

Fig. 1. Partial level diagram for O IV.

Table 1. O Iv spectral lines included in the present analysis.

\begin{tabular}{lll}
\hline \hline Instrument & Wavelength $(\AA)$ & Transition \\
\hline SUMER & 790.19 & $2 \mathrm{~s}^{2} 2 \mathrm{p}^{2} \mathrm{P}_{3 / 2}-2 \mathrm{~s} 2 \mathrm{p}^{2}{ }^{2} \mathrm{D}_{5 / 2}$ \\
SUMER & 790.11 & $2 \mathrm{~s}^{2} 2 \mathrm{p}^{2} \mathrm{P}_{3 / 2}-2 \mathrm{~s} 2 \mathrm{p}^{2}{ }^{2} \mathrm{D}_{3 / 2}$ \\
SUMER & 787.72 & $2 \mathrm{~s}^{2} 2 \mathrm{p}^{2} \mathrm{P}_{1 / 2}-2 \mathrm{~s} 2 \mathrm{p}^{2}{ }^{2} \mathrm{D}_{3 / 2}$ \\
CDS & 555.76 & $2 \mathrm{~s}^{2} 2 \mathrm{p}^{2} \mathrm{P}_{3 / 2}-2 \mathrm{~s} 2 \mathrm{p}^{2}{ }^{2} \mathrm{P}_{1 / 2}$ \\
CDS & 554.51 & $2 \mathrm{~s}^{2} 2 \mathrm{p}^{2} \mathrm{P}_{3 / 2}-2 \mathrm{~s} 2 \mathrm{p}^{2}{ }^{2} \mathrm{P}_{3 / 2}$ \\
CDS & 554.08 & $2 \mathrm{~s}^{2} \mathrm{p}^{2} \mathrm{P}_{1 / 2}-2 \mathrm{~s} 2 \mathrm{p}^{2}{ }^{2} \mathrm{P}_{1 / 2}$ \\
CDS & 553.33 & $2 \mathrm{~s}^{2} 2 \mathrm{p}^{2} \mathrm{P}_{1 / 2}-2 \mathrm{~s} 2 \mathrm{p}^{2}{ }^{2} \mathrm{P}_{3 / 2}$ \\
EIS & 279.93 & $2 \mathrm{~s}^{2} 2 \mathrm{p}^{2} \mathrm{P}_{3 / 2}-2 \mathrm{~s}^{2} 3 \mathrm{~s}^{2} \mathrm{~S}_{1 / 2}$ \\
EIS & 279.63 & $2 \mathrm{~s}^{2} 2 \mathrm{p}^{2} \mathrm{P}_{1 / 2}-2 \mathrm{~s}^{2} 3 \mathrm{~s}^{2} \mathrm{~S}_{1 / 2}$ \\
\hline
\end{tabular}

$R_{1}=I(787.72 \AA) / I(279.93 \AA)$ is re-examined and three other ratios are investigated:

- $R_{2}=I(554.51 \AA) / I(279.93 \AA)$;

$-R_{3}=I(787.72 \AA) / I(554.51 \AA) ;$

- $R_{4}=I(787.72 \AA) / I(b 790 \AA)$.

The $R_{2}$ and $R_{3}$ ratios involve the strongest line of the CDS multiplet, O Iv $554.51 \AA$, together with the EIS line at $279.93 \AA$ and the SUMER line at $787.72 \AA$ respectively. Both $R_{2}$ and $R_{3}$ are temperature sensitive, similarly to $R_{1} . R_{4}$ is the ratio of two SUMER lines, where $b 790$ is, as previously indicated, a selfblend of the two lines at $790.11 \AA$ and $790.19 \AA$.

It is noted that Muglach et al. (2010) repeated the study for pairs of $\mathrm{O}$ vi lines at $1037.61 \AA$ (observed by SUMER), $183.94 \AA$ and $184.12 \AA$ (observed by EIS), drawing similar conclusions. The $\mathrm{O}^{5+}$ ion is not included in the present analysis, since the key O vi SUMER doublet (at $1031.93 \AA$ and $1037.61 \AA$ ) was not available in the present observations.

In Sect. 2, the joint observations of April 2009 are described. The atomic data sources are discussed in Sect. 3. Sections 4 and 5 present the results and final conclusions.

\section{Observations and data analysis}

The present work explores a new data set obtained under Joint Observing Program 220 (JOP 220) and Hinode Operation Plan 109 (HOP 109). The instruments involved are SUMER (Wilhem et al. 1995) and CDS (Harrison et al. 1995) onboard the Solar and Heliospheric Observatory (SoHO) and EIS (Culhane et al. 2007) onboard Hinode. The joint observing campaign took place in April 2009. The data selected and analysed here were taken on 17 April from 12:00 to 16:00 UT. Instruments were pointed to a quiet Sun area near the Sun centre at $\left(0^{\prime \prime},-40^{\prime \prime}\right)$. The
Table 2. Details of the joint SUMER, CDS, EIS observations.

\begin{tabular}{lccc}
\hline \hline $\begin{array}{l}\text { Observing } \\
\text { parameters }\end{array}$ & SUMER & CDS & EIS \\
\hline Start & $12: 00 \mathrm{UT}$ & $12: 00 \mathrm{UT}$ & $12: 02 \mathrm{UT}$ \\
End & $15: 17 \mathrm{UT}$ & $16: 37 \mathrm{UT}$ & $15: 52 \mathrm{UT}$ \\
Pointing & $\left(0^{\prime \prime},-40^{\prime \prime}\right)$ & $\left(-1^{\prime \prime},-40^{\prime \prime}\right)$ & $\left(0.9^{\prime \prime},-26^{\prime \prime}\right)$ \\
Slit & $1^{\prime \prime} \times 120^{\prime \prime}$ & $2^{\prime \prime} \times 240^{\prime \prime}$ & $1^{\prime \prime}\left(/ 2^{\prime \prime}\right) \times 256^{\prime \prime}$ \\
FOV & $90^{\prime \prime} \times 120^{\prime \prime}$ & $80^{\prime \prime} \times 240^{\prime \prime}\left(/ 150^{\prime \prime}\right)$ & $60^{\prime \prime} \times 256^{\prime \prime}$ \\
Exp. time & $30 \mathrm{~s}$ & $55 \mathrm{~s}$ & $40 \mathrm{~s}$ \\
Windows & 19 & 14 & 7 \\
Scans & 2 & 6 & 9 \\
\hline
\end{tabular}

data were taken by SoHO/SUMER, SoHO/CDS normal incidence spectrometer (NIS) and Hinode/EIS, using $1 \times 120 \mathrm{arcsec}^{2}$, $2 \times 240 \operatorname{arcsec}^{2}$ and $1(/ 2) \times 256 \operatorname{arcsec}^{2}$ slits, respectively. The fields of view (FOV) covered by the three instruments were $90 \times 120 \operatorname{arcsec}^{2}$ for SUMER, $80 \times 240 \operatorname{arcsec}^{2}$ for CDS/NIS (except for its last scan which covers the area $80 \times 150 \operatorname{arcsec}^{2}$ ) and $60 \times 256 \operatorname{arcsec}^{2}$ for EIS. The exposure time at each location was $30 \mathrm{~s}$ for SUMER, $55 \mathrm{~s}$ for CDS and $40 \mathrm{~s}$ for EIS. These values were large enough to ensure a high signal-to-noise ratio in the observed fluxes. Table 2 summarises the main details of the joint observations. The coordinates are given in arcseconds, with the Sun's centre at $(0,0)$. Positive $X$-values are westward and positive $Y$-values are northward. Solar tracking was not used for CDS and EIS scans, while it was turned on for SUMER scans. However, because of the very quiet conditions during these observations, the general pattern of observed structures was very stable over extended periods of time. Moreover, we average line intensities over a common area observed by the three instruments. This reduces substantially the effects of small-scale and short-time fluctuations, which are an intrinsic characteristic of the transition region and lower corona.

The new SUMER, CDS and EIS observational sequences were designed to guarantee the spatial and temporal overlap between the instruments. Two raster maps were made with SUMER, by moving the slit in the east-west direction. The first scan includes selected bands with wavelengths shorter than the first line of the hydrogen Lyman series $\left(\lambda_{\mathrm{Ly}-\alpha}=1215.7 \AA\right)$, while the second scan includes the wavelength ranges greater than that wavelength. By contrast, six CDS and nine EIS scans were obtained by moving the slits in the west-east direction. Each scan contains the whole set of wavelength bands, selected for the CDS and EIS observation sequences. To mitigate the effects of the lack of completely co-temporal observations, all CDS and EIS rasters were averaged and then analysed with the two SUMER rasters.

Standard SUMER spectral data reduction was applied. The radiometric calibration was obtained using a procedure described by Curdt et al. (2001). It includes a correction factor of $43 \%$ to take into account the decrease of sensitivity due to the loss of SoHO in 1998.

The raw CDS/NIS data were reduced and calibrated using the standard procedures and routines within SolarSoft ${ }^{2}$, as described by Brooks et al. (1999) and Lang et al. (2002, 2007). However, the burn-in correction of the CDS/NIS detectors was recently revised by Del Zanna et al. (2010), providing new long term wavelength-dependent correction factors which give significant changes in the calibrated intensity, especially for the strongest lines, such as He I $584.33 \AA$ and O v $629.73 \AA$. Such

${ }^{2}$ http://www.lmsal.com/solarsoft/ 


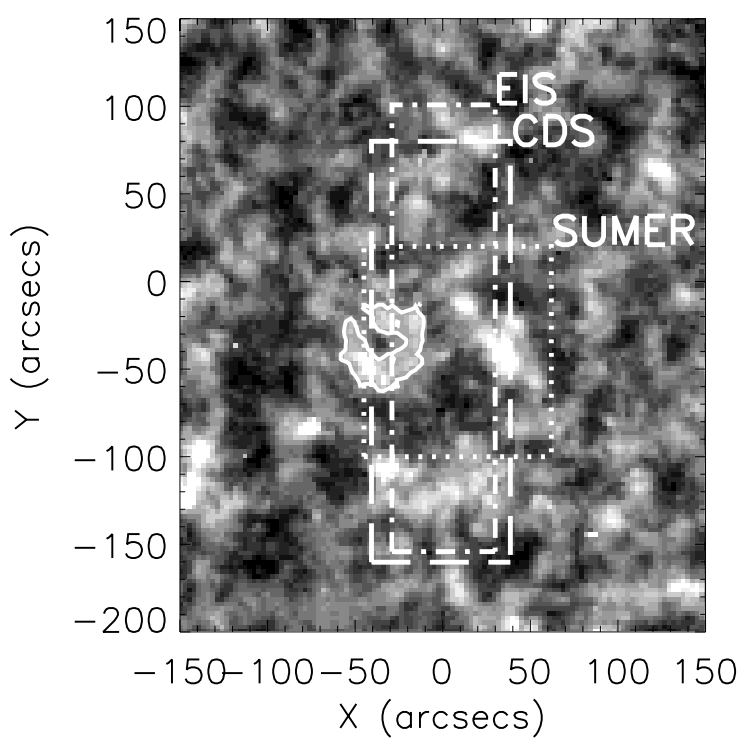

Fig. 2. Regions observed by the three spectrometers, SUMER, CDS and EIS, on 17 April 2009. The full disk image shows the Sun as observed in the He II $304 \AA$ band. It was obtained by the EUV Imaging Telescope (EIT) onboard SoHO on the same date at 08:19:17 UT. The field of view and the pointing of the SUMER, CDS and EIS slits are marked with different line styles, respectively dotted, dashed and dot-dashed lines. The continuous contour indicates the feature which is used for co-alignment in Fig. 3.

lines are included in the present observations and are very important for the co-alignment and cross-calibration between CDS and SUMER. These new correction factors were adopted in the present work. The uncertainty in the observed intensity was estimated to be between $15 \%$ and $30 \%$, essentially due to the burnin uncertainty and the uncertainties in the wavelength-dependent count to photon conversion. An additional $10 \%$ error was introduced for observations taken after SoHO's loss.

The reduction and calibration of the EIS datasets was done using the "eis_prep" routine available in the SolarSoft distribution, as described by Young et al. (2009). The accuracy of the EIS absolute intensity calibration was estimated to be around $20 \%$ before the launch (Lang et al. 2006).

The SUMER, CDS and EIS spectrometers were nominally pointed at the same location on the Sun, but the actual pointings were as indicated in Table 2. The pointing accuracy of SUMER and CDS is within 5 arcsec, while typical values of EIS pointing uncertainty are 5-10 arcsec. However, the fields of view covered by the three spectrometers are wide enough to allow a good overlap (Fig. 2) and to perform an appropriate coalignment. Note that the background image in Fig. 2 was taken at 08:20 $\mathrm{UT}^{3}$, while the present observations cover a time between 12:00 and 16:00 UT. As a consequence, because of the Sun's rotation, the feature specified by the continuous contour in Fig. 2 and used in the co-alignment procedure moved by about 40 arcsec from the left edge to the right edge of the common field of view, as shown in Fig. 3. Since the images taken by the three instruments are characterised by different spatial resolution along both $X$ and $Y$ axis, each image was resized such that it is comparable with the images recorded at lowest resolution in the set (which are the CDS images). This reduced spatial resolution is not a concern, because the present analysis deals with

\footnotetext{
3 This was the image from the EUV Imaging Telescope closest to our joint SUMER-CDS-EIS observations.
}
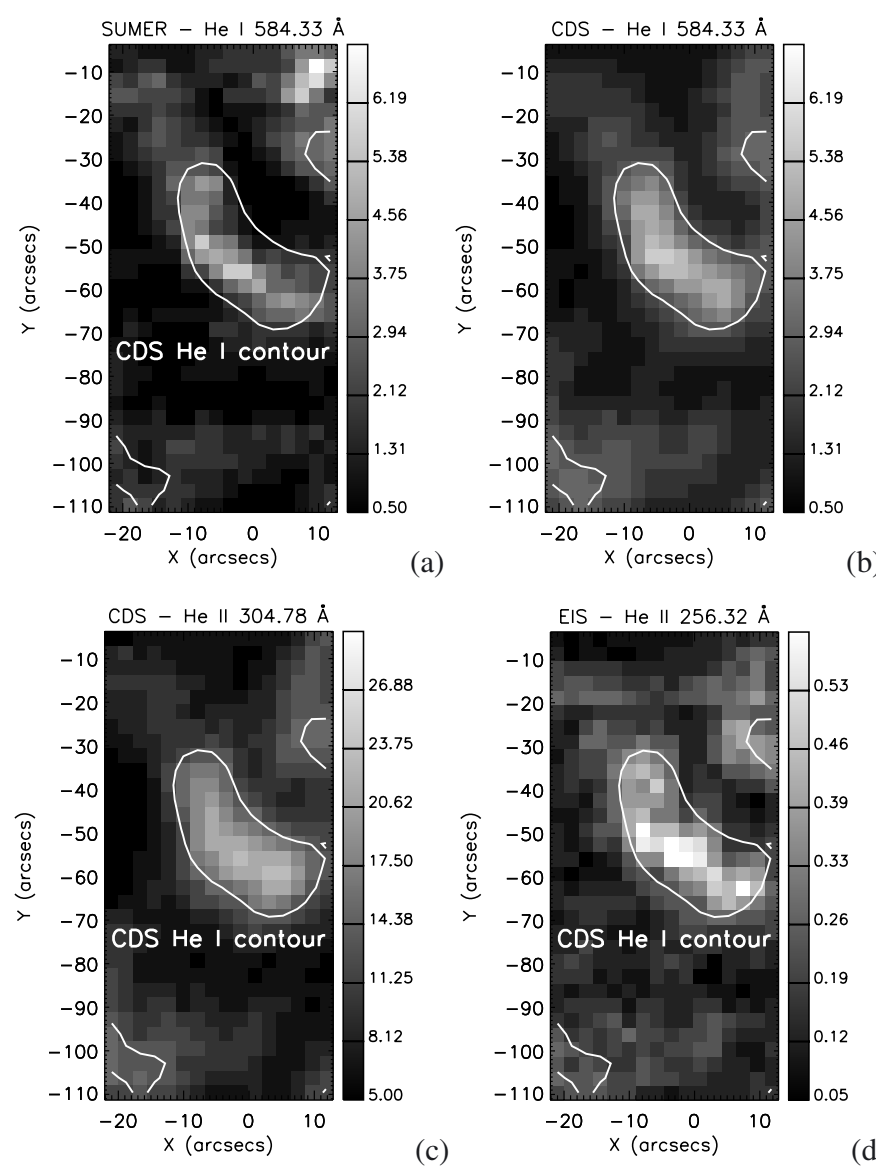

(b)

Fig. 3. Final cropped rasters of SUMER, CDS and EIS. He I $584.33 \AA$ line intensity distribution is shown for SUMER and CDS a) and b). He II $304.78 \AA$ and He II $256.32 \AA$ doublet intensity distributions are displayed in c) and d) respectively. The first one is observed by CDS, the second one by EIS. The white solid line overplotted on each image is the contour of CDS He I $584.33 \AA$ intensity. The intensity scale unit is $10^{13}$ photons $\mathrm{cm}^{-2} \mathrm{~s}^{-1} \mathrm{sr}^{-1}$.

averages along the two spatial dimensions of each raster. Once the spatial arrays have been rescaled, the co-alignment between the instruments has been carried out using lines or multiplets which originate from the same ions or which are close in formation temperature: He I $584.33 \AA$ (second order) for SUMER; He I $584.33 \AA$ and He II $304.78 \AA$ (second order) for CDS; He II $256.32 \AA$ for EIS. The latter He II doublet is blended with mainly Si x 256.37 $\AA$, Fe x 256.41 $\AA$ and Fe xIII 256.42 $\AA$, but, for disk observations, He II has been estimated to contribute 70$80 \%$ of the blend, so that it is still possible to use its intensity distribution to provide a reliable co-alignment. The co-alignment along north-south and east-west directions was performed using the "cross_corr2" routine, available within SolarSoft. The final cropped and co-aligned rasters are shown in Fig. 3.

Before applying the spectral fitting procedure, all observed spectra were averaged over the final common field of view. The method used to fit a theoretical spectrum is based on the program developed by Lang et al. (1990) and implemented as code ADAS602 within ADAS, as described by Brooks et al. (1999). The SUMER and EIS spectra were fitted with Gaussian line profiles and a linear background. For the CDS spectra, modified Gaussian profiles were adopted as developed by Thompson (1999), to take into account the post-recovery observed line 
Table 3. Comparison between SUMER and CDS line intensities (photons $\mathrm{cm}^{-2} \mathrm{~s}^{-1} \mathrm{sr}^{-1}$ ).

\begin{tabular}{lcccc}
\hline \hline Line & & $\begin{array}{c}\text { SUMER } \\
\text { intensity }\end{array}$ & $\begin{array}{c}\text { CDS } \\
\text { intensity }\end{array}$ & Ratio \\
\hline $\mathrm{He}_{\mathrm{I}}$ & $584.33 \AA$ & $1.7 \times 10^{13}$ & $1.9 \times 10^{13}$ & 0.9 \\
$\mathrm{O}_{\mathrm{v}}$ & $629.73 \AA$ & $1.4 \times 10^{13}$ & $1.3 \times 10^{13}$ & 1.1 \\
\hline
\end{tabular}

profiles (after the SoHO loss) which exhibited wings of different strength on each side.

Finally, since three different instruments are used, it is essential that their relative intensity calibration is accurate, to avoid systematic error in their line intensity comparison. The intercalibration between SUMER and CDS/NIS was thoroughly discussed by Pauluhn et al. $(1999,2001)$. They examined the intensities measured by the two instruments before and after the SoHO's attitude loss, including He r $584.33 \AA$, Mg x $609.79 \AA$ and $\mathrm{Mg}$ x $629.94 \AA$ lines in the pre-loss datasets and adding $\mathrm{O} v$ $629.73 \AA$ line in the post-loss datasets. They found that in the $\mathrm{He}$ I line at $584.33 \AA$ the CDS instrument measured $33-38 \%$ higher values than SUMER for the pre-loss period, while for the post-loss period the relative differences of the average intensity amounted to $43 \%$ for He I $584 \AA$ and $-4 \%$ for O v $629.73 \AA$. Here, the new burn-in correction of Del Zanna et al. (2010) has been applied for the absolute calibration of CDS. A 1.5 correction multiplier is used for present SUMER observations to allow for an 0.5 arcsec gap between successive 1 arcsec slit widths on the solar surface. The new comparison between the spatially averaged intensity of SUMER and CDS is given in Table 3 for the two common lines included in the present observations. The He I line intensity observed by CDS shows a value $10 \%$ higher than the SUMER value, while in the $\mathrm{O} v$ line the CDS instrument measures 7\% lower value than SUMER.

The relative intensity calibration between SUMER and EIS was determined recently by Landi \& Young (2010). Due to the lack of common emission lines, they established the SUMER-EIS relative calibration through spectroscopic diagnostics techniques, including Emissione Measure (EM), Differential Emission Measure (DEM) and line ratios. They concluded that a good agreement between the intensities observed by the two spectrometers was found when SUMER line intensities were multiplied by a $1.28 \pm 0.17$ correction factor, which accounts for the residual east-west misalignment of the two fields of view. This is comparable to the 1.5 correction factor assumed in this paper which is arrived at from different considerations. Additionally, the SUMER-CDS-EIS cross-calibration was checked by comparing line intensities emitted by ions belonging to the same element and calculating the DEM using each instrument separately. These further tests were needed to verify the adopted relative calibration among the three instruments.

\section{Atomic data}

The first 125 fine-structure energy levels, belonging to the configurations $2 \mathrm{~s}^{2} 2 \mathrm{p}, 2 \mathrm{~s} 2 \mathrm{p}^{2}, 2 \mathrm{p}^{3}, 2 \mathrm{~s}^{2} 31,2 \mathrm{p}^{2} 3 l$ and $2 \mathrm{~s} 2 \mathrm{p} 3 l$ with $l=$ $\mathrm{s}, \mathrm{p}, \mathrm{d}$, are included in the CHIANTI dataset for $\mathrm{O}^{3+}$. The experimental energy levels are taken from NIST (National Institute of Standards and Technology ${ }^{4}$ ) while the theoretical values are taken from the work of Zhang et al. (1994) and Sampson (1995, priv. comm.) The radiative transition probabilities

\footnotetext{
${ }^{4}$ http://www.nist.gov/
}

(A-values) for ground forbidden transitions have been taken from Flower \& Nussbaumer (1975), while for the allowed transitions $2 s^{2} 2 p-2 s 2 p^{2}$ and $2 s^{2} 2 p-2 s^{2} 3 s$ the values from Corrégé \& Hibbert (2004) are used. All others are taken from NIST. The effective collision strengths ( $(\Upsilon$-values) for 105 transitions among the first 15 levels have been computed using the R-matrix method, including fine structure and relativistic effects, by Zhang et al. (1994). For the remaining transitions the distorted-wave (DW) calculations of Sampson (1995, priv. comm.) have been adopted. However, only transitions which involve the first five levels belonging to the configurations $2 s^{2} 2 p$ and $2 \mathrm{~s} 2 \mathrm{p}^{2}$ and between them and the other 120 levels have been taken into account in CHIANTI. $A$-values for transitions amongst levels from 6 to 15 and all other higher levels and for transitions between $2 s^{2} 3 s^{2} S_{1 / 2}-2 s^{2} 3 p^{2} P_{1 / 2,3 / 2}$ (which are levels 16 and 73,87) have been included, but the corresponding $\Upsilon$-values are not present in the dataset.

Therefore, a revised dataset has been built up, merging the CHIANTI data with the DW calculations of Sampson collected in the ADAS database in order to include the whole set of missing collision strengths. This means that the radiative and collisional data for transitions involving the first five levels come from CHIANTI, while the data for transitions amongst all the other levels included in the model are taken from the Sampson (1995, priv. comm.) collection.

It is noted that there are more recent calculations for $\mathrm{O}^{3+}$ performed by Aggarwal \& Keenan (2008) which are discussed and used in next section. Their dataset includes 75 fine-structure energy levels belonging to the $2 \mathrm{~s}^{2} 2 \mathrm{p}, 2 \mathrm{~s} 2 \mathrm{p}^{2}, 2 \mathrm{p}^{3}, 2 \mathrm{~s}^{2} 3 l, 2 \mathrm{~s} 2 \mathrm{p} 3 l$ and $2 \mathrm{~s}^{2} 4 l^{\prime}$, with $l=\mathrm{s}, \mathrm{p}, \mathrm{d}$ and $l^{\prime}=\mathrm{s}, \mathrm{p}, \mathrm{d}, \mathrm{f}$, configurations. The energy levels and $A$-values were calculated using the fully relativistic code GRASP (General-purpose Relativistic Atomic Structure Package) and the $\Upsilon$-values using the fully relativistic DARC (Dirac Atomic R-matrix Code), providing data for 2775 transitions.

\section{Results and discussion}

Figure 4 a shows the comparison between observed and theoretical values of the ratio $R_{1}$, which involves the two $\mathrm{O}$ IV lines analysed by Muglach et al. (2010).

The observational points of April 2007 have been taken from Muglach et al. (2010) while the measurement of April 2009 has been derived as described in Sect. 2. The spread of all the observational points (cross and circles) is due to the different observed regions of the Sun in the 2007 and 2009 datasets as well as the different calibration factors adopted for SUMER in Muglach et al. (2010) and in the present work. The latter effect contributes to the spread by $15 \%$. The ratio $R_{1}$ does not depend on electron density, as discussed by Muglach et al. (2010), so the electron density has been fixed at $10^{10} \mathrm{~cm}^{-3}$ hereafter. However this line ratio does depend on the electron temperature, as shown in Fig. 4a. For the theoretical ratio of Muglach et al. (2010), derived from CHIANTI, the measured points agree with the theoretical values in the electron temperature range of $\log T=5.58-6.07$. If either our revised atomic data or the Aggarwal \& Keenan data are adopted instead, the electron temperature range for the April 2007 measurements changes to $\log T=5.29-5.39$ and is even lower when the April 2009 observational point $(\log T=$ $5.17)$ is included. These values are much closer to the $O$ IV formation temperature, suggesting that the main discrepancy lies in the atomic data. Note that the temperature of maximum fractional abundance in ionisation equilibrium has been adopted here as the $\mathrm{O}$ IV $\left(\mathrm{O}^{3+}\right.$ ion $)$ formation temperature to be consistent with 
A. S. Giunta et al.: $\mathrm{O}$ Iv line ratios in the solar UV/EUV spectrum
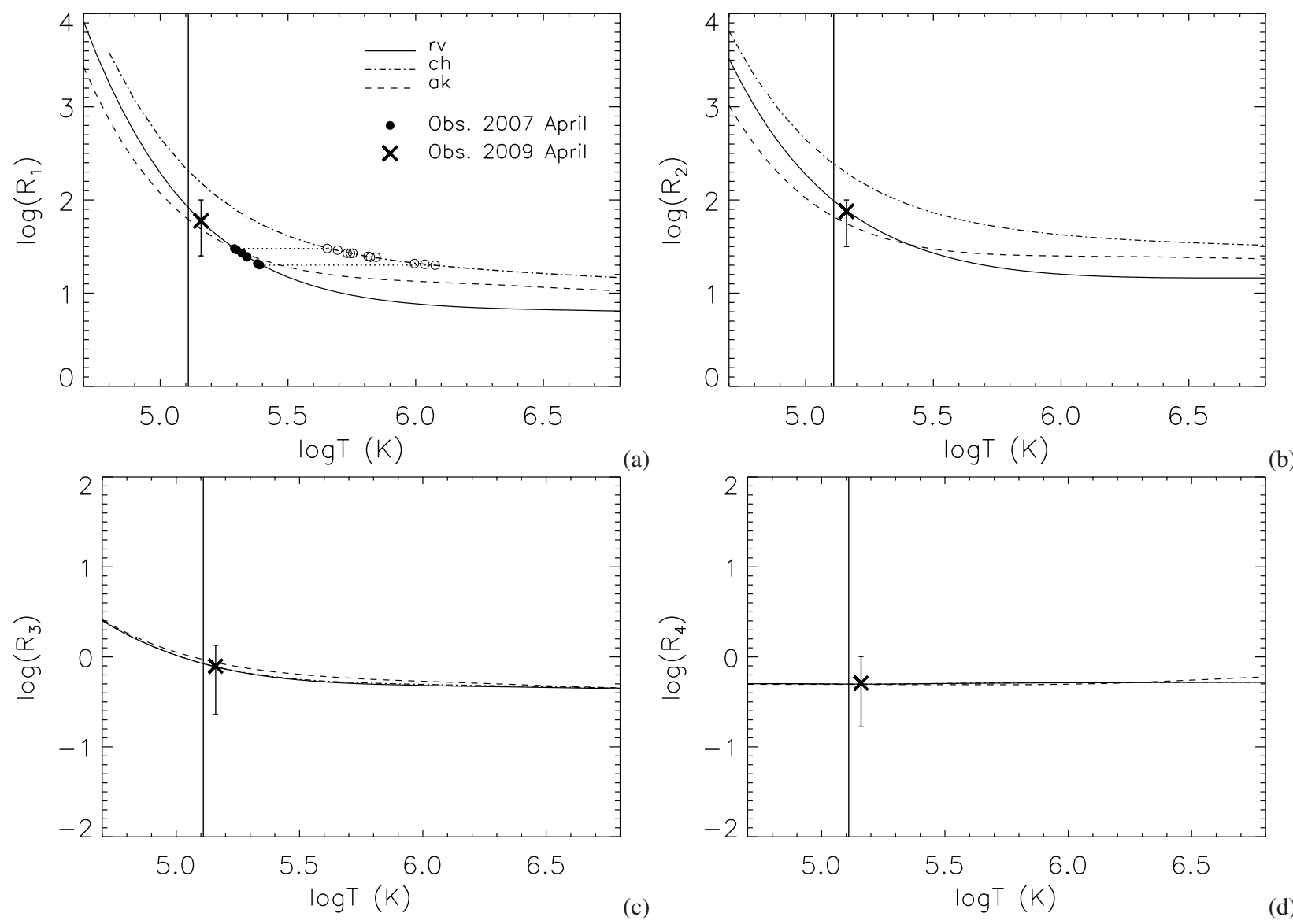

b)

Fig. 4. Comparison between the theoretical and measured ratios of $\mathrm{O}$ iv: a) $R_{1} ;$ b) $R_{2} ;$ c) $R_{3}$; d) $R_{4}$. The theoretical ratios have been obtained from the three datasets: CHIANTI, denoted as ch; revised, denoted as rv; and Aggarwal \& Keenan (2008), denoted as ak. The filled circles indicate the observations of April 2007 taken from Muglach et al. (2010), while the crosses, with the error bars, represent the observations of April 2009. The open circles show where the observational points of April 2007 must be plotted if the CHIANTI data are adopted as in Muglach et al. (2010). The intensity units are photons $\mathrm{cm}^{-3} \mathrm{~s}^{-1}$. The adopted electron density is $N_{\mathrm{e}}=10^{10} \mathrm{~cm}^{-3}$. The vertical lines are at the $\mathrm{O}$ Iv temperature formation, $\log T_{\mathrm{m}}=5.11$. Note that the ch, rv and ak data are basically identical for the ratios $R_{3}$ and $R_{4}$ shown in panels $\mathbf{c}$ ) and d).

Muglach et al. (2010), although strictly the formation temperature is a little higher, determined from the peak of the line contribution function ${ }^{5}$. For instance, at $N_{\mathrm{e}}=10^{10} \mathrm{~cm}^{-3}$, the contribution function of the line at $787.72 \AA$ peaks at $\log T_{\mathrm{m}}=5.15$, while the contribution function of the line at $279.93 \AA$ peaks at $\log T_{\mathrm{m}}=5.25$.

Since both emission lines arise from the same ion, their intensity ratio can be written as the emissivity ratio at fixed values of electron temperature and electron density:

$\frac{I(787.72 \AA)}{I(279.93 \AA)}=\frac{\varepsilon_{787}}{\varepsilon_{279}}=\frac{A_{787} N_{787}}{A_{279} N_{279}}$

where $\varepsilon_{787}, \varepsilon_{279}$ are the emissivities of the two emission lines considered, $A_{787}, A_{279}$ are the radiative transition probabilities related to the two transitions and $N_{787}, N_{279}$ are the population densities of the upper level of each transition, i.e. $2 s 2 p^{2}{ }^{2} D_{3 / 2}$ and $2 s^{2} 3 s^{2} S_{1 / 2}$ respectively.

As discussed in Sect. 3, the difference between the CHIANTI and revised datasets is the number of transitions included. The truncation of the CHIANTI dataset affects essentially the population of the $2 \mathrm{~s}^{2} 3 \mathrm{~s}^{2} \mathrm{~S}_{1 / 2}$ level, $N_{279}$. The difference arises

\footnotetext{
5 A line contribution function is defined as the product of the photon emissivity coefficient, which describes the population of excited levels, and the fractional abundance.
}

from the contribution due to the radiative decay from the term $2 \mathrm{~s}^{2} 3 \mathrm{p}^{2} \mathrm{P}^{\mathrm{o}}$. The population of the two levels belonging to this term is underestimated by $63-73 \%$ in CHIANTI, depending on the electron temperature. As a consequence $N_{279}^{\text {ch }}<$ $N_{279}^{\mathrm{rv}}$ by a factor $2-3$ while $N_{787}^{\mathrm{ch}} \sim N_{787}^{\mathrm{rv}}$, where ch indicate the CHIANTI dataset and rv the revised dataset. Therefore $\varepsilon_{787}^{\text {ch }} / \varepsilon_{279}^{\text {ch }}>\varepsilon_{787}^{\text {rv }} / \varepsilon_{279}^{\text {rv }}$ by a factor $2-3$ and the CHIANTI ratio is higher than the revised ratio, as shown in Fig. 4a.

In principle, this is sufficient to explain the disagreement between the observed and predicted values for the $I(787.72 \AA) / I(279.93 \AA)$ line ratio, found in the Muglach et al. (2010) paper. However, as a further check the present revised dataset (rv) has been compared with the recent calculation of Aggarwal \& Keenan (2008) (ak).

It is evident from Fig. 4a that in the region of $\mathrm{O}$ IV formation $\left(\log T_{\mathrm{m}}=5.11\right)$, the difference between the two datasets (rv and ak) is within a few percent, but it increases at lower and higher temperature by $40-50 \%$. The main difference lies in the collisional excitation rate coefficients, which populate the $2 s^{2} 3 s^{2} S_{1 / 2}$ level, and in the population of $2 s^{2} 3 p^{2} \mathrm{P}^{\mathrm{o}}$ term. The Aggarwal \& Keenan collisional excitation rate coefficients are larger than those included in the revised data by $85 \%$ at $\log T=4.80$ and this difference decreases to $20 \%$ as the electron temperature increases. On the other hand, the population of $2 \mathrm{~s}^{2} 3 \mathrm{p}^{2} \mathrm{P}^{\mathrm{o}}$ term from Aggarwal \& Keenan data is lower by 
$60-75 \%$ (depending on electron temperature) compared to the population from the revised data. The different proportions between these two contributions in different temperature regimes explains the difference between the revised and Aggarwal \& Keenan datasets. In spite of the divergence at low and high temperatures, the Aggarwal \& Keenan ratio and our revised ratio are much closer to the observed ratio than the CHIANTI ratio, demostrating the importance of using a complete system of $A$ values and effective collision strengths.

As a further check, the three other $\mathrm{O}$ IV line ratios, $R_{2}, R_{3}$ and $R_{4}$, have been analysed, comparing the measurements with the theoretical ratios derived from the CHIANTI, revised and Aggarwal \& Keenan datasets. Since the difference is caused by the population of the level $2 s^{2} 3 s^{2} S_{1 / 2}$, it is expected that the $R_{3}$ and $R_{4}$ theoretical ratios should agree with the measurements. The $R_{2}$ ratio should show the same behaviour as the $R_{1}$ using the three datasets. These findings are illustrated in Figs. $4 \mathrm{~b}-\mathrm{d}$ and furthermore the electron temperature derived from the three temperature dependent ratios $R_{1}, R_{2}$ and $R_{3}$ is the same $(\sim \log T=5.17)$, reflecting the consistency of the atomic dataset used.

\section{Conclusions}

We have obtained new measurements of $\mathrm{O}$ IV using joint observation by SUMER, CDS and EIS and compared both the new observations and the previous observations by Muglach et al. (2010) to new theoretical line ratios. Our improved calculation has been verified using three other line ratios originating from the same ion and available in the SUMER, CDS and EIS wavelength bands. We have solved the discrepancy of a factor 2-5 for the O IV $I(787.72 \AA) / I(279.93 \AA)$ line ratio, reported by Muglach et al. (2010). The source of the inconsistency has been found to be the incomplete atomic data used to calculate the intensity of the O IV $279.93 \AA$ line. The electron temperature derived from all three $\mathrm{O}$ Iv line ratios gives consistent results providing a temperature range $\log T=5.17-5.39$, close to the temperature expected under the assumption of the ionisation equilibrium.

Acknowledgements. This work has been supported by the University of Strathclyde and the UK Science and Technology Facilities Council. The authors thank the SUMER, CDS and EIS teams for providing new observational sequences and scheduling the observations. The authors thank Dr. Werner Curdt for help in the SUMER calibration.

\section{References}

Aggarwal, K. M., \& Keenan, F. P. 2008, A\&A, 486, 1053

Brooks, D. H., Fischbacher, G. A., Fludra, A., et al. 1999, A\&A, 347, 277

Corrégé, G., \& Hibbert, A. 2004, ADNDT, 86, 19

Culhane, J. L., Harra, L. K., James, A. M., et al. 2007, Sol. Phys., 243, 19

Curdt, W., Brekke, P., Feldman, U., et al. 2001, A\&A, 375, 591

Del Zanna, G., Andretta, V., Chamberlin, P. C., Woods, T. N., \& Thompson, W. T. 2010, A\&A, 518, A49

Dere, K. P., Landi, E., Young, P. R., et al. 2009, A\&A, 498, 915

Flower, D. R., \& Nussbaumer, H. 1975, Sol. Phys., 45, 145

Harrison, R. A., Sawyer, E. C., Carter, M. K., et al. 1995, Sol. Phys., 162, 233

Landi, E., \& Young, P. R. 2010, ApJ, 714, 636

Landi, E., Del Zanna, G., Young, P. R., et al. 2006, ApJS, 162, 261

Lang, J., Mason, H. E., \& McWhirter, R. W. P. 1990, Sol. Phys., 129, 31

Lang, J., Kent, B. J., Paustian, W., et al. 2006, Appl. Opt., 45, 8689

Lang, J., Brooks, D., Lanzafame, A., et al. 2007, A\&A, 463, 339

Lang, J., Thompson, W. T., Pike, C. D., Kent, B. J., \& Foley, C. R. 2002, in The Radiometric Calibration of SOHO. ISSI Scientific Report SR-002, ed. A. Pauluhn, M. C. E. Huber, \& R. von Steiger, 105

Mazzotta, P., Mazzitelli, G., Colafrancesco, S., \& Vittorio, N. 1998, A\&ASS, 133,403

Muglach, K., Landi, E., \& Doschek, G. A. 2010, ApJ, 708, 550

Pauluhn, A., Rüedi, I., Solanki, S. K., et al. 1999, Appl. Opt., 38, 7035

Pauluhn, A., Rüedi, I., Solanki, S. K., et al. 2001, Appl. Opt., 34, 6292

Summers, H. P. 2001, The ADAS manual, version 2.3, http://adas.ac.uk

Wilhem, K., Curdt, W., Marsch, E., et al. 1995, Sol. Phys., 162, 189

Young, P. R., Watanabe, T., Hara, H., \& Mariska, J. T. 2009, A\&A, 495, 587

Zhang, H. L., Graziani, M., \& Pradhan, A. K. 1994, A\&A, 283, 319 